De la migration à la mobilité : changement de paradigme dans la gestion migratoire. Le cas du Canada

From Migration to Mobility: Paradigm Change in Migration. The Canadian Case De la migración hacia la movilidad: cambio de paradigma en la migración. El caso de Canadá

\title{
Hélène Pellerin
}

\section{(2) OpenEdition}

Journals

Édition électronique

URL : https://journals.openedition.org/remi/5435

DOI : $10.4000 /$ remi. 5435

ISSN : $1777-5418$

Éditeur

Université de Poitiers

Édition imprimée

Date de publication : 1 octobre 2011

Pagination : $57-75$

ISBN : 979-10-90426-01-6

ISSN : 0765-0752

Référence électronique

Hélène Pellerin, « De la migration à la mobilité : changement de paradigme dans la gestion migratoire. Le cas du Canada », Revue européenne des migrations internationales [En ligne], vol. 27 - n² | 2011, mis en ligne le 01 octobre 2014, consulté le 14 avril 2022. URL : http://journals.openedition.org/remi/5435 ; DOI : https://doi.org/10.4000/remi.5435 


\title{
De la migration à la mobilité : changement de paradigme dans la gestion migratoire. Le cas du Canada
}

\author{
Hélène PELLERIN ${ }^{1}$
}

\section{INTRODUCTION}

T a Commission globale sur la migration internationale chargée de faire le point

ـur les tendances et les stratégies les plus appropriées pour les États a évoqué l'apparition d'un nouveau paradigme de la migration, axé sur la migration temporaire et circulaire (GCIM, 2005). L'Organisation Internationale pour les Migrations (OIM) a tenu des propos similaires dans un récent rapport annuel, en soulignant que « la quête d'un emploi sera au cœur de la plupart des mouvements migratoires du XXIe siècle » (OIM, 2008). Ces tendances, qui varient énormément d'un pays industrialisé à l'autre, contrastent pourtant avec les initiatives développées en Europe, en Australie et en Amérique du Nord au cours des quinze dernières années précédentes visant à mieux contrôler les flux migratoires, voire à les endiguer.

Depuis quelques années l'étude de la migration rend compte de cette mutation contrastée dans les politiques. Quelques études amorcent une réflexion sur la migration comme objet de gouvernance, insistant sur les pratiques de recrutement tout autant que celles visant à limiter les flux. Elles font état de nouvelles modalités de contrôles et de recrutement des migrants à travers des ententes bilatérales ou régionales (Ghosh, 2000 ; Tamas et Palm, 2006) et dans le cadre des accords commerciaux (Pellerin, 2008 ; Panizzon, 2010). Les modalités du mouvement seraient également changeantes, avec l'existence de migrations temporaires ou circulaires, de retour temporaire ou définitif, soulignant le recours croissant à la main-d'œuvre étrangère (Martin, Abella et Kuptsch, 2006).

1 Professeure agrégée, École d'études politiques, Université d'Ottawa, 55 ave. Laurier est, Ottawa, Ontario, K1N6N5, Canada ; hpelleri@uottawa.ca 
Dans ce contexte, il convient de souligner la substitution progressive du concept de migration par celui de mobilité. Cette référence conceptuelle est devenue fréquente autant dans les sphères politiques que dans la recherche. Des économistes évoquent la mobilité des personnes comme faisant partie des forces de la mondialisation (Bhagwati, 2003). Le concept de mobilité semble servir à décrire de nouvelles modalités de mouvement et d'insertion des étrangers, à savoir les migrations circulaires et temporaires et des conditions inédites de gestion migratoire. Les pays les plus industrialisés, qui sont les plus touchés par ces transformations, deviendraient des États migratoires (Hollifield, 2004). Pour certains, ce serait le commencement d'une mutation profonde de la gestion migratoire ; d'un paradigme de la migration, nous serions passés à un paradigme de la mobilité (Papademetriou, 2009). Même des chercheurs traitant des enjeux de sécurité relatifs à la migration soulignent que c'est la mobilité internationale, et non la migration internationale, qui pose le plus de risques aujourd'hui (Koslowsky, 2004 : 6).

Si la mobilité s'impose comme cadre conceptuel en remplacement du terme de migration, il faut pouvoir en saisir les implications, car plus qu'un constat, elle constitue également un discours et un paradigme, renvoyant alors à un modèle économique, social et politique de gestion du travail et de la place du travail dans la société. Afin d'explorer ces propositions, l'article qui suit procédera à l'étude des tendances migratoires dans les pays industrialisés, pour ensuite analyser le processus par lequel un modèle de mobilité et de sa gestion se met en place, avec de nouvelles catégories administratives, des acteurs et des modalités d'intervention qui s'imposent et remplacent les références à la migration. Et puisque les transformations discursives sont liées à des changements socio-économiques importants, l'analyse de la mobilité sera remise dans son contexte, celui d'une transformation de l'économie des pays industrialisés et du rôle de l'État. Le cas du Canada servira à illustrer les implications de ces mutations dans la gestion migratoire.

\section{L'IMPORTANCE DE LA MOBILITÉ AUJOURD'HUI}

Depuis le début des années 2000, le concept de mobilité s'est imposé pour décrire des nouvelles formes de migration dans le contexte de la mondialisation. L'année de son lancement en 2006, le Dialogue de Haut Niveau sur la Migration et le Développement a fait de la mobilité de la main-d'œuvre une stratégie centrale de croissance. Dans son Rapport 2008 sur l'état de la migration dans le monde, l'Organisation Internationale pour la Migration indique que « la mobilité humaine s'est imposée comme choix de vie dicté par les disparités en termes de démographie, de revenus et d'opportunités d'emploi entre les régions et en leur sein » (OIM, 2008).

La référence à la mobilité reflète en grande partie des politiques migratoires spécifiques destinées à recruter des travailleurs et un contexte politico-économique issu d'une décennie de néo-libéralisme. Cette mobilité serait la conséquence directe de la libéralisation des flux de capitaux, des biens et des services. Elle touche plus particulièrement les pays industrialisés et certaines catégories de migrants. C'est ainsi que la mobilité des travailleurs hautement qualifiés est liée à la croissance du secteur tertiaire dans les pays développés, à l'importance accrue du commerce des services favorisée par l'Accord Général sur le Commerce des Services (AGCS) et aux nombreux accords régionaux de 
commerce, aux changements technologiques et à la transnationalisation de la production (Lavenex, 2008 ; Pellerin, 2008). Pour ce qui est des flux de main-d'œuvre peu qualifiée, ils sont encouragés par le maintien sinon l'aggravation d'écarts de richesse entre pays pauvres et pays riches qui agissent comme facteur de répulsion. Dans les pays industrialisés, le vieillissement de la population et des besoins criants de main-d'œuvre, voire de manœuvres dans des secteurs géographiquement fixes ne pouvant être délocalisés, assurent des besoins constants. Dans les pays plus pauvres, on adopte des stratégies visant à promouvoir une plus grande mobilité internationale, destinée aux pays riches.

Ce qu'il faut retenir de ces tendances c'est que la mobilité d'aujourd'hui serait encouragée par les États et les organisations internationales. L'Organisation Internationale pour les Migrations fait la promotion d'accords de circulation migratoire, notamment pour gérer les besoins de travail dans le secteur agricole. Dans son compendium des meilleures pratiques en matière de gestion, l'OIM évoque d'ailleurs la nécessité pour les États de mettre en place toutes les mesures qui puissent garantir que les migrations temporaires ne se transformeront pas en migrations permanentes (OIM, 2005). Les États industrialisés ont, pour la plupart, adopté des politiques de recrutement de travailleurs temporaires, non seulement pour les migrants peu spécialisés, mais aussi pour la main-d'œuvre hautement qualifiée. Pour le travail non qualifié, on favorise la migration circulaire, sous forme de contrats d'emploi temporaire variés et de sous-traitance. Les travailleurs hautement qualifiés sont recrutés par une politique d'immigration axée sur le capital humain, des contrats de travail associés aux besoins sur le marché du travail, au recrutement d'entrepreneurs, et enfin à travers l'admission d'étudiants étrangers dans les universités (Abella, 2006).

Ces tendances dans les politiques s'accompagnent d'une substitution progressive du concept de migration par celui de mobilité. Dans le tout nouveau glossaire de l'UNESCO, censé rendre compte de l'évolution de la terminologie associée à la migration internationale, la mobilité est définie comme la catégorie générale servant à décrire tout déplacement géographique de personnes, peu importent la durée, la composition ou les motivations sous-jacentes au mouvement (Meyer et Witkapm, 2008). La mobilité couvrirait donc aussi bien les déplacements de vacanciers que la migration et elle s'appliquerait autant aux déplacements internationaux qu'aux mouvements à l'intérieur d'une seule juridiction nationale. Sur le plan purement technique, son adoption pour parler de migration fait référence à des distinctions assez précises dans l'espace, la durée et dans les motivations selon au moins trois traits caractéristiques. Il y a d'abord la courte durée de la migration, entre trois et douze mois selon les recommandations de l'ONU (Auriol et Sexton, 2001). Contrairement à la migration qui serait sur le long terme et définitive, la mobilité serait éphémère et circulaire. Une deuxième caractéristique porte sur l'élargissement des catégories de personnes mobiles. Dans son Glossaire de la migration, l'Organisation Internationale pour les Migrations définit la mobilité comme processus englobant « tous les types de mouvements de population impliquant un changement du lieu de résidence habituelle, quelles que soient leur cause, leur composition, leur durée, incluant ainsi notamment les mouvements des travailleurs, des réfugiés, des personnes déplacées ou déracinées », nous dit Perruchoud (2007). La mobilité représenterait un certain élargissement des possibilités d'entrée et de séjour dans un pays d'accueil, des visas d'études en passant par les contrats de travail à durée limitée et des transferts internationaux à l'inté- 
rieur d'une firme, en passant par la fourniture de services à l'étranger (Favell, Feldblum et Smith, 2008). Mais simultanément, et c'est là le troisième critère, le terme correspondrait à un contexte économique distinct, différencié des conditions historiques dans lesquelles la migration fut encouragée tant dans les pays d'origine que dans les pays d'accueil au cours des années d'après-guerre. Wallace posa très bien cette distinction lorsqu'elle nota que le terme de mobilité était plus propice pour rendre compte de la migration dans un contexte post-industriel dans lequel il n'y aurait pas nécessairement de correspondance entre l'offre et la demande de travailleurs étrangers (Wallace, 2002).

\section{LA MOBILITÉ COMME CADRE CONCEPTUEL}

\section{La mobilité, modèle et réalité sociale}

Dans les années 1970, le terme de mobilité était rarement employé pour parler de migration internationale. La mobilité était plutôt réservée aux mouvements à l'intérieur des frontières, alors que le terme de migration faisait référence aux mouvements internationaux de personnes (Fortes, 1971). Le terme de migration était associé à la période de croissance industrielle, aux migrations de travailleurs gérées par l'offre et la demande sur le marché du travail (Wallace, 2004), ainsi qu'au règne du principe de souveraineté qui octroyait à tous les États le monopole du pouvoir de contrôle sur les flux transfrontaliers (Smith et Favell, 2008). La mobilité serait plutôt associée directement au libéralisme, et particulièrement au libéralisme économique, sans friction générée par les interventions étatiques.

Géographes et sociologues ont introduit le concept pour évoquer la fluidité des rapports sociaux qui touche l'ensemble de la société. Elle serait facilitée entre autres par les développements technologiques et le progrès des communications et des moyens de transport (Urry, 2007). Mais la mobilité doit aussi être présentée dans son articulation avec la société. Il y aurait, selon Kaufmann (2002) plusieurs conceptions de la mobilité, déclinées selon son importance relative dans une société. Le modèle auréolaire serait le plus limité, subordonnant la mobilité aux lieux, aux frontières et autres mécanismes de la fixité sociale. Le modèle réseautisé, est caractérisé par la mobilité de certains éléments de la société rattachés à des réseaux transnationaux. Le modèle liquide ferait de la mobilité la caractéristique dominante d'une société, englobant tous les phénomènes sociaux. La conception rhizomatique -plus un idéal qu'une réalité - conçoit un monde totalement fluide où disparaissent les distinctions entre l'interne et l'externe et la nécessité de mobilité (Kaufman, 2002 : 7-9). Seul le premier modèle correspondrait à une période historique précise, l'ère pré-industrielle durant laquelle les personnes étaient fixées au sol par des conditions économiques, politiques et idéologiques particulières. Les trois autres modèles constituent des idéaux, associés à des formes de mobilité particulière et projetées par des intérêts particuliers.

Ces diverses conceptions mettent en valeur le caractère profondément social de la mobilité ; c'est en relation avec la société que la mobilité prend son sens. Mais pour mieux comprendre les assises historiques et concrètes de ces modèles, il convient d'analyser 
le contexte social plus large dans lequel s'inscrivent le mouvement des personnes et sa gestion. Comme le soulignait Bourdieu, le terrain du langage seul ne suffit pas à expliquer les luttes et leur issue. Le contexte socio-politique et économique constitue lui aussi un terrain de lutte de ces visions.

\section{Entre discours et contexte : le paradigme de la mobilité}

C'est aux travaux de Noiriel (2008 et 2001) et d'autres historiens de l'immigration que l'on doit d'avoir problématisé les mots de l'immigration, pour en souligner le pouvoir performatif. Larbiou (2003 et 2008) parle ainsi d'une matrice migratoire pour saisir le sens particulier de la migration d'une époque et qui structure les politiques migratoires. Selon cette approche, la mobilité ne serait pas le reflet d'une réalité migratoire nouvelle, naturellement associée à l'économie mondialisée ou aux stratégies des États qui cherchent ainsi à moduler la migration déjà présente en fonction de la concurrence internationale. Le terme sert plutôt à présenter une certaine cohérence entre la migration et le contexte socio-économique plus large et à comprendre comment celle-ci s'est développée. Sur le plan analytique et suivant les travaux de Noiriel et Larbiou, on peut appeler paradigme ce contexte dans lequel la migration et sa gestion se réalisent. En tant que concept heuristique, le paradigme représente un cadre englobant, visant à faciliter la mise en commun des analyses fragmentées sur les stratégies des migrants, sur les politiques de gestion et sur le contexte économique de la migration dans un ensemble qui développerait une certaine cohérence, non pas naturellement et inexorablement, mais par le travail délibéré d'experts et d'instances bureaucratiques. La notion de paradigme permet d'identifier les principales composantes d'un paradigme migratoire, à savoir la matrice ou discours dominant, les politiques de gestion migratoire qui l'accompagnent et les rapports de forces économiques sur lesquels elle s'appuie. Elle ne cherche pas à établir une causalité définitive entre l'économique, le politique et la migration, mais plutôt à saisir dans un ensemble constitutif, les influences réciproques. La notion de paradigme migratoire permet de saisir comment une vision devient dominante et supplante les autres. Le paradigme est plus que la représentation d'une dynamique migratoire dans une période donnée, car en produisant des politiques et des catégories migratoires, il se constitue en matrice qui exerce une influence sur l'univers des possibilités des États, sur les pratiques exemplaires de la part des entreprises et même sur les stratégies des migrants.

Toute période historique ayant ses particularités, il faut pouvoir distinguer entre différents paradigmes migratoires. La prépondérance de certains intérêts économiques ou politiques ou encore l'existence de liens de sociabilité particuliers donne lieu à des dynamiques distinctes, que l'analyse socio-historique peut révéler. Noiriel parlait par exemple d'un paradigme de la trace pour évoquer l'origine du problème de l'immigration en France au XIXe siècle. L'évocation de ce paradigme sert à mettre l'accent sur les transformations administratives et politiques ayant conduit au développement d'une matrice générale englobant le phénomène migratoire. Le paradigme de la trace évoque l'émergence de la catégorie administrative de l'étranger en France au cours du XIXe siècle et ses liens avec le développement de mesures de contrôles des déplacements et du suivi des sujets et des objets ayant franchi la frontière. Pour parler de la gestion migratoire dans l'après-guerre, Larbiou (2008) suggère le concept de paradigme de la production. 
Pour cet auteur, la référence au paradigme de la production sert à mettre l'accent sur la centralité des activités productives et du travail dans la constitution et la gestion des flux migratoires, avec l'implication d'agences d'employeurs qui étaient restées, jusque-là, à l'écart de la gestion migratoire. Et dans le cadre du paradigme de la production, la matrice donnant cohérence au système était celle du développement économique et de la gestion de la main-d'œuvre. Les pays industrialisés ont alors fait du recrutement de migrants l'une des stratégies de développement industriel. Les besoins de main-d'œuvre définis par les employeurs et approuvés par les gouvernements ont contribué à produire un discours et une certaine cohérence nationale autour de la migration comme un facteur de développement économique, envisagé dans un horizon national et endogène. L'équation entre migration et développement économique était ensuite relayée par des accords migratoires entre États d'origine et États d'accueil. Les questions de traçabilité, de sécurité ou du statut d'étranger étaient reléguées au second rang. Sans être éliminées, ces préoccupations sur la surveillance des étrangers ont alors été réarticulées en fonction de la matrice de production, avec des réglementations sur le travail et sur les divers statuts de travailleurs.

Dans le paradigme de la mobilité émergeant, on retrouve, aux côtés des acteurs plus traditionnels que sont les employeurs et les organisations syndicales, des associations d'entreprises axées sur les stratégies commerciales et qui utilisent la mobilité des étrangers pour restructurer l'offre de travail plutôt que pour répondre à une demande existante. La mobilité fait alors partie des stratégies d'expansion économique. Les entreprises privées participent à la construction d'une matrice qu' ils entretiennent à travers une production de rapports d'experts et de services de consultation auprès des gouvernements. Les migrants eux-mêmes peuvent participer à sa construction, dans la mesure où ils sont capables de déployer des stratégies de mobilité qui les avantagent ; ce qui correspond en l'occurrence aux activités des migrants hautement qualifiés et des gens d'affaires. Les moins qualifiés adoptent plutôt des stratégies de survie dans une situation qu'ils subissent plus qu'ils ne la maîtrisent.

La dimension performative du narratif de la mobilité s'exerce essentiellement à trois niveaux. D'abord du point de vue des politiques liées aux modalités d'entrée et de contrôle des flux migratoires. Dans la pratique des politiques migratoires, le terme mobilité devient souvent synonyme de migration temporaire et de la multiplication des modes de migration. Diverses catégories de migration temporaire sont favorisées, car en plus des travailleurs temporaires, on compte les étudiants, les investisseurs et les entrepreneurs, avec des statuts et des traitements différenciés selon qu'il s'agisse de visas d'études, de transferts, de contrats à durée déterminée ou encore de fournisseurs de service. L'orientation plus exogène de l'économie fait aussi en sorte que la migration est gérée beaucoup plus directement au travers d'ententes commerciales et de développement économique à l'échelle régionale. Ce narratif suggère également une nouvelle façon de comprendre la migration, en tant que fluidité dans la circulation des personnes, éphémère et surtout, réversible. Ceci marque une rupture par rapport aux catégories des travailleurs rattachées à des postes précis dans le paradigme de la production. Et devant les enjeux d'exode de cerveaux, les experts soulignent les nouvelles complémentarités entre les besoins des pays industrialisés en main-d'œuvre étrangère et en termes de croissance démographique d'une part et la tendance irrévocable à une plus grande mobilité des personnes touchant en particulier les pays d'origine d'autre part. 
C'est ainsi que la mobilité recherchée peut se situer soit en amont du processus, comme c'est le cas pour les travailleurs peu qualifiés, que l'on exhorte à être mobiles. Des États d'origine, comme le Maroc, encouragent d'ailleurs la création de viviers de candidats à l'émigration, afin de répondre dans les temps, à une demande de travail à l'étranger. Mais du même coup, on fixe par contrat les travailleurs à des employeurs ou à des emplois précis, sans possibilité de dérogation. La mobilité en aval du processus de migration est donc très contrôlée pour les non qualifiés, car les employeurs veulent « fixer l'homme au sol » (Morrice et Michalon, 2008). Plusieurs analystes ont d'ailleurs dénoncé les pratiques abusives et discriminatoires des migrations temporaires (Shamir, 2005 ; Sassen, 1998 ; Vertovec, 2007 ; Morice et Michalon, 2008). Dans les secteurs à haute densité de capital humain, la référence à la mobilité est plus générale et sert à mieux attirer le capital humain. Car dans ce secteur, sans doute le plus intégré mondialement, la mobilité se trouve autant en amont qu'en aval des sites de travail ; et les notions de rareté et de surplus associées aux travailleurs hautement qualifiés depuis la mise en œuvre de l'Accord Général sur le Commerce des Services en indiquent l'existence (Lavenex, 2008). Il y aurait aussi une pression exercée pour créer les conditions propices à la mobilité du capital humain, notamment en facilitant la standardisation des compétences, ou en offrant des conditions fiscales avantageuses dans les pays d'accueil, ou encore en favorisant la migration ordonnée dans les pays d'origine. Il ne s'agirait pas d'abandonner les préoccupations de sécurité et de rentabilité dans une politique d'ouverture tous azimuts ${ }^{2}$, mais de promouvoir une bonne mobilité. Pour y parvenir, les États envisageraient ou avaliseraient des modalités différenciées de mobilité, selon les catégories de personnes. C'est donc dire que les préoccupations de sécurité et de traçabilité seraient réorientées vers la production de régimes différenciés de mobilité des personnes.

L'action performative s'exerce aussi à l'endroit des individus. La mobilité serait une stratégie collective de développement économique axé sur l'investissement social et la recherche d'une plus grande productivité de l'ensemble des interlocuteurs. La mobilité engloberait autant les stratégies des natifs que des étrangers, et autant des travailleurs rattachés à des professions spécifiques que des étudiants avec des compétences particulières. Dans le langage des experts, la mobilité constitue la situation la plus propice pour atteindre l'optimisation des bénéfices. Elle établit un lien presque naturel entre l'économie mondialisée et le mouvement (Borjas, 1989 ; Bhagwati, 2003), en suggérant que la mobilité aurait été déclenchée par la mondialisation, une sorte de verrou qui tombe et qui laisse libre court au mouvement naturel des individus. Le concept de mobilité tend à promouvoir autant l'idée de la normalité du mouvement - et l'on s'évertue à montrer comment la migration est une constante dans l'histoire de l'humanité et qu'elle favorise des situations bénéfiques pour tous - que la continuité logique entre la mobilité sociale et la mobilité géographique. La catégorie de la mobilité contiendrait l'expérience générale de l'homo economicus, dans la mesure où elle deviendrait la stratégie universelle de promotion sociale en incitant du même coup ces individus mobiles à développer leurs réseaux sociaux par eux-mêmes. Cette vision fait de la mobilité un mécanisme d'allocation ou de distribution d'un facteur de production dans l'espace ; elle devient un moyen servant une fin, celle d'une distribution optimale dans l'économie. La mobilité équivaut au

2 Très évocateur à cet égard est le nombre de concepts répertoriés dans le glossaire de l'UNESCO reliés aux préoccupations de sécurité des États : migrants irréguliers, sans papier, illégaux, clandestins, sans autorisation, ou migrants sur des épaves (2008). 
déplacement des individus, vus comme des « éléments individuels post-sociaux, atomisés et en apesanteur, mus par les forces du marché mondial » (Meyer, Kaplan et Charum, 2001 : 342). Suivant une logique fonctionnaliste, la mobilité pourrait même servir à empêcher des situations de crise de se développer sur la base des trois « $\mathrm{D}$ » de l'ordre mondial actuel, à savoir les écarts Démographiques, Démocratiques et de Développement (Adepoju, 2006). La nouvelle matrice se construit sur la double idée de promotion de la mobilité et de production de modes ordonnés de mobilité pouvant créer un nouveau capital social susceptible d'être bénéfique pour l'économie mondiale et les migrants (Geyer et Straubhaar, 2005).

Troisièmement, le narratif de la mobilité exerce un effet performatif sur les acteurs économiques dans leur ensemble. Dans ce paradigme, le mouvement dépasse les besoins du marché du travail national. Au cœur de la matrice se retrouvent des considérations de stratégies de croissance, de position concurrentielle et de compétences flexibles et transversales, supplantant les calculs et demandes axées exclusivement sur les besoins en main-d'œuvre définis selon des postes spécifiques, comme c'était le cas dans le paradigme de la production. L'effet performatif s'exerce sur les entreprises, en offrant une nouvelle légitimité aux stratégies de croissance axées sur l'échange et la circulation. Car dans la nouvelle théorie de la croissance, nous disent les experts, la productivité dépend des effets d'agglomération et des externalités positives et négatives, c'est-à-dire de la capacité à concentrer la production dans des lieux précis et complémentaires les uns des autres. L'externalisation sert à exclure de ses coûts les activités non rentables et à favoriser une plus grande spécialisation. La mobilité des travailleurs constitue un atout central dans cette optique, car elle facilite la concentration et la spécialisation dans des lieux localisés. La mobilité sert aussi, et c'est là le propre du nouveau contexte économique, de stratégie de croissance et de développement des capacités des entreprises. Tel est le cas notamment dans les secteurs de l'économie du savoir, comme les télécommunications et l'informatique ; secteurs où la mobilité de la main-d'œuvre se réalise à l'intérieur même des firmes, sans avoir recours à l'État. La flexibilité géographique des travailleurs hautement qualifiés permet d'opérer des stratégies de spécialisation dans lesquelles les régions du monde deviennent soit complémentaires soit concurrentielles les unes par rapport aux autres. Même le domaine de l'agriculture adopte ce genre de stratégie, puisque dans les pays industrialisés, les mesures d'agglomération ont permis la modernisation du secteur et son positionnement concurrentiel sur les marchés mondiaux. Il convient de souligner combien cette stratégie d'agglomération permet de distinguer le paradigme de la mobilité de celui de la production. Dans ce dernier, c'était la recherche de coûts de production plus faibles qui guidait les stratégies des entreprises et qui définissait la matrice migratoire. La migration permettait de réduire les coûts de production et de flexibiliser le travail. Dans le paradigme de la mobilité, c'est surtout dans la recherche de nouvelles stratégies de concurrence ou de productivité que la migration est envisagée.

Le tableau qui suit vise à poser de manière contrastée les caractéristiques des deux principaux paradigmes du XXe siècle. Certes, cet effort analytique présente des généralisations qui gomment au passage les nuances nationales et surtout le caractère inachevé des paradigmes. Dans le paradigme de la production par exemple, il s'est avéré que nombreuses ont été les entrées qui n'étaient pas reliées au travail, comme, par exemple l'immigration par regroupement familial ou encore les demandeurs d'asile qui 
se sont poursuivis à divers degrés dans les pays industrialisés ${ }^{3}$. De même, le paradigme de la mobilité ne se substitue pas entièrement au précédent, laissant encore des instances de migration de travail, comme on le voit par exemple dans le domaine de la construction immobilière ou celui de certaines professions où c'est plus la recherche de moindres coûts qui amène les firmes et les États à la sous-traitance. Mais le tableau a le mérite de camper de manière succincte les conditions historiques de la migration.

Tableau 1 : Tableau analytique des paradigmes migratoires du XXe siècle

\begin{tabular}{|c|c|c|}
\hline Paradigme migratoire & De la production & De la mobilité \\
\hline Acteurs & $\begin{array}{l}\text { Agences gouvernementales associées } \\
\text { à l'industrie } \\
\text { Employeurs } \\
\text { Syndicats } \\
\text { Pays d'origine (à travers ententes } \\
\text { bilatérales) }\end{array}$ & $\begin{array}{l}\text { Agences gouvernementales associées } \\
\text { à la formation de la main-d'œuvre, à } \\
\text { l'éducation } \\
\text { Secteur du commerce } \\
\text { Associations de gens d'affaires } \\
\text { Diasporas }\end{array}$ \\
\hline Modes d'entrée & $\begin{array}{l}\text { Contrats de travail } \\
\text { Immigration économique }\end{array}$ & $\begin{array}{l}\text { Immigration économique } \\
\text { Contrats de travail } \\
\text { Visas d'études } \\
\text { Transferts } \\
\text { Visas d'investisseurs }\end{array}$ \\
\hline $\begin{array}{l}\text { Catégories migratoires } \\
\text { principales }\end{array}$ & $\begin{array}{l}\text { Travailleurs (aussi regroupement } \\
\text { familial et demandeurs d'asile }\end{array}$ & $\begin{array}{l}\text { Capital humain : } \\
\text { Étudiants } \\
\text { Travailleurs } \\
\text { Fournisseurs de services } \\
\end{array}$ \\
\hline Durée du séjour & Permanent ou temporaire & Courte durée mais pouvant se répéter \\
\hline Matrice & $\begin{array}{l}\text { La production et gestion du marché } \\
\text { du travail }\end{array}$ & $\begin{array}{l}\text { Recherche de capital humain Création } \\
\text { de capital social }\end{array}$ \\
\hline
\end{tabular}

\section{L'ÉMERGENCE DU PARADIGME DE LA MOBILITÉ AU CANADA}

\section{Tendances statistiques et politiques}

Prendre le Canada comme exemple peut paraître paradoxal, car il s'agit d'un pays ayant une longue tradition d'immigration d'installation et où le recours à la migration de courte durée peut paraître exceptionnel. Par ailleurs, pour les observateurs de la situation canadienne, parler de paradigme dans le domaine migratoire peut sembler exagéré tant les politiques ont toujours manqué de cohérence et de vision d'ensemble. L'étude des politiques migratoires depuis l'après-guerre leur donne partiellement raison. Le Canada s'est bien doté d'un système migratoire permettant de sélectionner et de recruter des immigrants

3 Au Canada, les candidats à l'immigration n'étaient pas toujours sélectionnés en fonction de leur correspondance avec le marché du travail. Au Canada l'autorisation de travail était fondée sur deux principes : protéger les travailleurs canadiens et résidents et donner aux employeurs canadiens l'accès aux compétences non disponibles sur le marché canadien. En pratique toutefois, le système était tel que les autorisations ont surtout été octroyées à des personnes ayant souvent peu à voir avec les besoins des employeurs (demandeurs d'asile, exemptés, candidats à l'immigration) (Boyd, Taylor et Delaney, 1982). 
pour des raisons démographiques et économiques ; mais paradoxalement, la présence d'un paradigme de la production cohérent est difficile à cerner pour cette époque. Plusieurs ont critiqué l'incapacité du système à répondre aux besoins du marché du travail, ainsi que son inefficacité causée par le retard accumulé dans le traitement des demandes d'immigration (Boyd, Taylor et Delaney, 1982 ; De Voretz, 2006 ; Reitz, 2005).

Depuis quelques années, plusieurs indices laissent croire à l'émergence du paradigme de la mobilité au Canada. On retrouve ainsi des politiques spécifiques de recrutement temporaire d'étrangers. La hausse, légère au début des années 2000, connaît une progression fulgurante par la suite, de $47 \%$ entre 2004 et 2007 (CIC, 2005 et 2008b). Et, fait encore plus notable, depuis 2006, l'entrée de travailleurs temporaires dépasse celle des immigrants économiques ${ }^{4}$. Ils étaient 139268 en 2006, comparativement à 138252 pour les immigrants permanents dans la classe économique ; 165215 en 2007, par rapport à 131244 ; et 193061 par rapport à 149047 (CIC, 2008a).

Cette tendance résulte d'une politique délibérée de préférence pour la migration temporaire. Le gouvernement conservateur de Stephen Harper l'a clairement indiqué dans sa stratégie économique en 2006. Dans son initiative Avantage Canada. Bâtir une économie forte pour les Canadiens, des prescriptions sont élaborées pour accroître la mobilité : « Nos politiques en matière d'immigration doivent concorder davantage avec les besoins observés sur le marché du travail. Un recours accru au Programme des candidats des provinces pourrait aider à combler les pénuries locales de main-d'œuvre. Il faudrait accorder une attention particulière aux travailleurs temporaires qui sont qualifiés et ont une expérience de travail au Canada de même qu'aux diplômés étrangers de nos collèges et universités, puisque ces personnes sont à même de s'adapter rapidement à l'économie canadienne » (ministère des Finances, Canada, 2006).

Au Canada, la migration temporaire liée au travail se retrouve dans de nombreux programmes distincts selon les critères d'admission, la réglementation sectorielle de l'emploi ou selon le cadre institutionnel de l'entente. Pour simplifier, on peut les diviser en deux catégories : les programmes pour les travailleurs peu qualifiés et ceux qui concernent les travailleurs hautement qualifiés ${ }^{5}$. Dans la catégorie des travailleurs hautement qualifiés, deux modalités existent : le programme pour les travailleurs en technologie de l'information et les emplois n'exigeant pas de permis de travail, notamment les emplois pour étudiants étrangers ou des visiteurs d'affaires. Le Canada a d'ailleurs développé une nouvelle voie d'immigration à partir de la catégorie de l'expérience canadienne, spécifiquement destinée aux étudiants étrangers et à quelques travailleurs temporaires qualifiés, les invitant à présenter une demande de résidence permanente à partir du Canada (CIC, 2008c).

Dans le cas de la migration de travailleurs non spécialisés, trois programmes existent : le programme des aides familiaux résidants, les programmes de travailleurs

4 Il convient de préciser que les statistiques mesurent le nombre d'entrées et non le nombre de travailleurs en situation temporaire, certains pouvant entrer plus d'une fois au cours d'une année.

5 La migration temporaire compte aussi des catégories non liées spécifiquement au travail comme les étudiants, les demandeurs d'asile et autres étrangers ayant une autorisation de séjour temporaire. 
agricoles saisonniers (PTAS) et un programme de travailleurs temporaires peu spécialisés. Il convient de souligner l'ascension fulgurante des PTAS au cours des dernières années. Ceux-ci, issus d'ententes entre le Canada et quelques pays des Caraïbes et du Mexique depuis le milieu des années 1960 et avec le Guatemala depuis $2003^{6}$, ont vu le nombre de migrants croître dans des proportions gigantesques ces dernières années. Le PTAS recrutait 264 travailleurs en 1966 et près de 20000 en 2006, avec deux périodes de croissance significative, au milieu des années 1980 d'abord, et à partir de 1995 par la suite (Preibisch, 2007).

Le Canada a aussi modifié son approche par rapport à la sélection d'immigrants permanents. Apparaissent depuis une vingtaine d'années de nouvelles catégories de migration associées à l'économie mais non directement au travail, en l'occurrence les migrants investisseurs et les compétences générales y compris le niveau d'éducation. Au cœur de cette approche généralisée à l'ensemble des pays développés ${ }^{7}$, se trouve la recherche de compétences. Une telle orientation aurait déjà été présente au Canada bien avant selon De Voretz (2006), car au moment de l'implantation du système de points pour la sélection des immigrants économiques en 1967, le niveau d'éducation et de compétences était au cœur du système. Mais suite aux pressions des employeurs, le système fut adapté pour mieux répondre aux besoins directs sur le marché du travail. Aujourd'hui, la recherche de compétence n'est pas déterminée selon les besoins précis du marché du travail, mais plutôt dans la perspective d'augmenter le bassin de connaissance dans la société canadienne. Le recrutement se détache des besoins du marché du travail afin d'anticiper et de produire un bassin de main-d'œuvre qualifiée susceptible d'accroître la compétitivité du Canada dans des domaines ciblés. Ceci a permis de créer des effets d'agglomération, avec par exemple de fortes concentrations d'employés nés à l'étranger dans les industries de haute technologie (Hall, 2006) et les professions de la santé (Bourgeault, 2006). Dans une comparaison de la situation du Canada à celle d'autres grands pays de l'OCDE, Industrie Canada affirme qu'autour de l'an 2000, le Canada a exceptionnellement bien réussi à attirer des immigrants permanents hautement scolarisés par rapport à d'autres pays concurrents, mais moins bien les travailleurs temporaires qualifiés et les étudiants internationaux de cycles supérieurs (Industrie Canada, 2008 : 10).

\section{La formulation d'une matrice de la mobilité}

Ce qui permet de parler de l'émergence d'un paradigme de la mobilité, c'est aussi l'existence d'un ensemble de référents idéologiques et stratégiques - une matrice qui contextualise et informe les politiques d'immigration. Les changements de politiques,

6 Des ententes furent signées avec la Jamaïque en 1966, la Barbade en 1967, Trinidad et Tobago en 1967 et le Mexique en 1974. (Preibisch, $2007: 423$ ).

7 Des données de l'OCDE indiquent que la recherche de compétences est au centre des nouvelles politiques de recrutement de la plupart des pays industrialisés. Depuis les années 1990, les tendances montrent une intensification marquée des flux de travailleurs hautement scolarisés de l'Asie vers des pays développés et une augmentation des échanges de travailleurs qualifiés parmi les pays industrialisés (Gera, Laryea et Songsakul, 2004). Et toujours selon l'OCDE, les principaux pays de l'OCDE, notamment les États-Unis, le Canada, l'Australie, la France, le RoyaumeUni et l'Allemagne, sont des importateurs nets de travailleurs hautement scolarisés (Dumont et Lemaître, 2005). 
en ce sens, ne seraient pas simplement ad hoc ou motivés par des intérêts particuliers passagers. La matrice de la mobilité s'insère, au Canada, dans un contexte socio-économique plus large, celui de la croissance post-industrielle et d'une approche plus large de politiques publiques, à savoir le modèle de l'État investisseur social. Ce modèle, issu du mouvement de la troisième voie théorisé par Anthony Giddens (Jenson et Saint-Martin, 2003), propose une nouvelle orientation pour les États développés, entre le laisser-faire néolibéral et l'État-providence. Contrairement au néolibéralisme, l'État reconnaît les limites du marché auto-régulateur et contrairement à l'État-providence, il ne cherche pas à protéger les citoyens $d u$ marché, mais plutôt à faciliter leur intégration et leur bonne performance dans le marché (Dobrowolsky, 2005). Les politiques publiques constituent des possibilités d'investissement dans la société et particulièrement dans le capital humain.

La gestion migratoire s'adapte à ce nouveau cadre, en poursuivant une politique qui favorise une meilleure utilisation du capital humain, qu'il soit étranger ou natif et une approche plus ouverte à l'intégration mondiale des marchés. C'est ainsi que dans son initiative " Avantage Canada. Bâtir une économie forte pour les Canadiens » en 2006, le gouvernement conservateur identifiait les avantages sur lesquels le gouvernement devait compter pour maintenir " un Canada fort dans un monde en évolution ». La mobilité des personnes constituait l'une des «trois vérités fondamentales » énoncées par le gouvernement. Les deux autres, à savoir les compétences comme atout principal du succès économique d'un pays et la nécessité d'instaurer un climat propice aux affaires, étaient déjà les pierres angulaires de la révolution néolibérale des années 1980 et 1990. Elles visaient la déréglementation et la croissance d'une économie du savoir. La stratégie sur la mobilité vient donner une nouvelle fonction aux politiques d'immigration. Pour devenir une composante utile et efficace, la migration doit être mieux régulée, c'est-à-dire que la mobilité des personnes doit être plus structurellement rattachée aux dynamiques économiques que ce n'est le cas actuellement. L'État canadien, représenté ici par les ministères de l'Industrie et celui des Ressources humaines et Développement social, a d'ailleurs fait siennes les conclusions d'un économiste qui disait : «Les répercussions d'une plus grande mobilité de la main-d'œuvre sur la dynamique de la productivité d'un pays est largement tributaire d'externalités localisées liées au capital humain, aux externalités du savoir au niveau mondial et aux économies de la spécialisation qu'il est davantage possible de réaliser dans un contexte de marchés du travail étroitement intégrés » (Industrie Canada, Ressources humaines et Développement social et CRSH, 2008).

Or, la gestion migratoire doit servir à réguler à la fois en amont et en aval les flux de personnes pour y arriver. Indépendamment de ce que les spécialistes des questions de sécurité en disent, les flux migratoires composés de migrants économiques restent assez peu réglementés une fois ceux-ci arrivés au Canada. Un analyste déclarait d'ailleurs que les flux migratoires constituaient encore aujourd'hui, dans les cas où la migration est permise, le facteur de production le moins réglementé de l'économie (Agunias, 2009). Une meilleure gestion migratoire consiste en ce sens, à instaurer, faciliter et réglementer les multiples voies de mobilité qui sont bénéfiques à l'économie d'aujourd'hui et de demain. Cela se traduit, par exemple, par l'établissement de postes d'observation dans certaines ambassades, c'est-à-dire dans les régions où le recrutement de capital humain serait avantageux, en amont du processus. Ce genre de services, existe déjà à l'ambassade canadienne à Pretoria en Afrique du Sud et est fortement encouragé par certains 
secteurs de l'économie, qui souhaiteraient voir se multiplier ces initiatives. Le Conference Board du Canada, une organisation indépendante promouvant les intérêts des entreprises, voudrait voir ce genre de services dans plusieurs pays émergents. En aval, la recherche de personnes hautement qualifiées est une stratégie déployée par plusieurs entreprises, bien que celles-ci aient encore du mal à garder ces compétences. Une étude récente montre qu'environ un tiers des immigrants masculins a quitté le pays durant les vingt premières années et que $60 \%$ de ceux qui ont quitté l'ont fait durant la première année suivant leur arrivée (Aydemir et Robinson, 2006). Plus problématique encore, les taux d'immigration seraient plus élevés pour les travailleurs qualifiés ou ceux qui détiennent un visa de gens d'affaires. Pour eux on estime à $40 \%$ les gens qui sont partis dix ans après leur arrivée (Aydemir et Robinson, 2006). Des données plus récentes permettent d'ailleurs de constater une décroissance de la proportion de travailleurs étrangers ayant des compétences de niveau C (niveau intermédiaire) et A (niveau professionnel) entre 2002 et 2007 (CIC, 2008b).

Pour ce qui est des travailleurs migrants non qualifiés, et en l'occurrence pour les travailleurs agricoles saisonniers, le recrutement s'est constitué comme stratégie de développement de l'industrie horticole d'exportation au Canada. Ceci vaut notamment durant la deuxième période de croissance du programme, à partir de 1995. C'est ainsi que le recours à la main-d'œuvre étrangère ne visait pas à répondre à une demande non satisfaite sur le marché canadien, mais plutôt à développer l'industrie d'exportation. Le secteur a ainsi diversifié ses produits : de production céréalière, on est passé à la production d'une large variété de légumes et de fruits. La présence de travailleurs étrangers a aussi permis d'étendre la saison avec la production en serres, et a permis au secteur agricole de devenir un exportateur net depuis cette période (Preibisch, 2007). La recherche d'agglomération et d'externalités s'est manifestée ici par la constitution d'un secteur capable de concurrencer efficacement l'agriculture américaine, déjà structurée de la sorte.

Ces transformations ont donné au secteur privé une plus grande place dans la gestion de la migration. Le paradigme de la mobilité produit donc une nouvelle relation entre le public et le privé, selon la perspective de l'État investisseur social. Le secteur privé intervient autant en amont qu'en aval de la gestion migratoire. On voit d'abord la place du secteur privé augmenter en amont, dans le traitement des modalités de recrutement des migrants temporaires. C'est le cas notamment dans les secteurs liés à l'économie du savoir. Car il s'agit-là d'un secteur où les entreprises fonctionnent déjà en mode intégré sur le plan continental ou mondial. Cela a facilité la mobilité géographique de la main-d'œuvre hautement qualifiée directement, sans passer par le recrutement avec des politiques d'immigration. L'État, dans ce cas, se trouve à la remorque du secteur, agissant comme facilitateur des mécanismes de mobilité. L'État canadien a approuvé par exemple la création d'agences spécialisées dans la normalisation des compétences pour répondre aux besoins de l'économie canadienne et aux engagements du Canada dans le processus de normalisation des compétences de Canberra (Auriol et Sexton, 2001). Plusieurs organismes publics et parapublics tels que l'Alliance canadienne des services d'évaluation de diplômes et l'Alliance des conseils sectoriels travaillent avec les associations privées comme la Fédération canadienne de l'entreprise indépendante et la Chambre de commerce du Canada, afin de déployer des modes de reconnaissance et des stratégies afin d'augmenter le rendement canadien en matière de recrutement et d'insertion de 
migrants hautement qualifiés. L'implication du secteur privé est également évidente en ce qui concerne les travailleurs peu qualifiés, notamment dans la procédure d'obtention de permis de travail. L'assouplissement des procédures pour obtenir les avis sur le marché du travail - ces permis nécessaires pour faire venir des travailleurs étrangers, émis par le ministère des Ressources humaines et du Développement des compétences - a permis de lier plus directement et plus rapidement les employeurs, les recruteurs et les travailleurs. Et cela est sans compter tous les types d'emploi déjà exemptés de la procédure.

On retrouve aussi une plus grande présence du secteur privé en aval du processus, en l'occurrence dans la gestion de programmes temporaires. Certains organismes privés à but non lucratif comme FERME (Fondation des Entreprises pour le Recrutement de la Main-d'œuvre Étrangère), FARMS et WALI (Western Agricultural Labour Initiative) gèrent directement les programmes (Brem, 2006). Une situation semblable touche les migrants hautement qualifiés, puisque l'on convient que dans la mesure où $80 \%$ des emplois sont non réglementés, c'est au secteur privé, c'est-à-dire aux employeurs, que revient la responsabilité de reconnaître les compétences (BORTCE, 2008).

La matrice de la mobilité qui émerge au Canada place donc le secteur privé au centre de la production du consensus social, relayé par certaines agences gouvernementales qui cherchent à définir et réglementer les nouvelles voies de la mobilité. Les efforts consentis jusqu'à présent ont surtout porté sur les modalités d'entrée, de séjour et de permis de travail des migrants. Ces politiques ont mené à la redéfinition progressive des catégories de l'immigration : le migrant économique, catégorie traditionnelle pour tous les travailleurs étrangers, s'estompe progressivement pour laisser place à de nouvelles distinctions entre les professionnels et les travailleurs hautement qualifiés et les étudiants étrangers, ainsi que le statut de travailleurs temporaires pour les autres migrants économiques sans compétences particulières.

\section{POUR CONCLURE}

De nombreuses analyses constructivistes de la migration furent proposées au cours des dix dernières années pour souligner la façon dont la migration est devenue délibérément un problème à gérer, voire une menace à la sécurité (Martiniello et Simon, 2005 ; Bigo, 1998 ; Noiriel, 2008). Celles-ci ont permis de tourner le projecteur de l'analyste vers les pratiques de ceux qui « d'en haut forgent ces catégories et contribuent toujours ainsi à la construction sociale d'un problème de l'immigration » (Hmed et Laurens, 2008 : 8). Ces analyses, qui ont le mérite de révéler les narratifs sous-jacents à des catégories et à des politiques spécifiques relatives à la population étrangère, sont particulièrement pertinentes pour dénaturaliser le phénomène de la mobilité internationale. L'approche proposée ici permet d'approfondir cette analyse en situant les formes de gestion migratoire et leurs registres conceptuels dans leur contexte économique et social. Ceci permet de voir comment la mobilité s'inscrit comme une réarticulation d'un projet de croissance économique. La restitution de l'évolution de la gestion migratoire d'un paradigme de la production, axé sur la composante économie industrielle, à la gestion migratoire économie post-industrielle, permet de documenter ce phénomène. 
La perspective historique du concept de paradigme migratoire comporte aussi l'idée d'une variété de mobilités et de degrés différenciés de mutation relatifs à des conditions socio-économiques nationales ou régionales. On peut supposer par exemple que l'émergence d'un paradigme de mobilité en Europe est assujettie à des défis propres à l'histoire de l'intégration européenne, à la distinction juridique entre citoyens européens et étrangers et à la quasi-absence institutionnelle des étrangers dans l'historiographie des pays européens. C'est donc dire que le paradigme de la mobilité s'implante à des degrés et des rythmes variés d'une région à l'autre.

On ne pourrait conclure sans mentionner quelques implications que la réflexion critique autour du paradigme de la mobilité permet de révéler. Le coût de cette mobilité élevée au rang de pratique exemplaire mérite d'être soulevé, car il dépasse nettement les enjeux d'ouverture et de fermeture des frontières. La vision idyllique d'une mobilité parfaite tirée par l'économie tranche avec la réalité sociale. En amont du processus migratoire, on note ainsi la promotion de situations de mobilité différenciées ; une mobilité temporaire dans les pays d'accueil, mais latente et permanente pour les personnes se situant dans les pays d'origine. C'est ainsi que l'on peut caractériser la création de ces « viviers » de candidats à la migration, toujours prêts à partir (Morice et Michalon, 2008). Cette réalité existe au Maroc et aux Philippines ${ }^{8}$ et est mise de l'avant par les États d'origine. Dans le cas du Mexique, les programmes de travailleurs temporaires signés avec le Canada obligeraient d'ailleurs la constitution de réservoir de candidats prêts à prendre la relève dans l'éventualité de ruptures de contrats par d'autres migrants déjà partis. À cela se rajoutent les coûts liés aux préparatifs à l'émigration - comme l'examen médical ou les visas - et qui sont absorbés par les candidats et les pays d'origine, sans même une garantie qu'ils puissent partir (Brem, 2006). La présence d'agences de recrutement censées gérer le marché de la mobilité constitue un autre problème dans le paradigme de la mobilité. Ces agences, qui se sont multipliées devant la demande croissante pour un capital humain mobile, sont peu réglementées. Selon une étude de l'Organisation Internationale du Travail, les contrôles de ces agences relèvent, comme au Canada, de la responsabilité du secteur privé lui-même (Agunias, 2009).

En aval du processus, c'est dans les sociabilités de la mobilité, dont le coût est absorbé presque totalement par les individus et le secteur privé, que le paradigme de la mobilité pose problème. Car le paradigme de la mobilité ne prévoit pas de mesures d'aide sociale spécifiquement pour les personnes mobiles. Il est vrai que de nouvelles formes de sociabilités se créent, au gré des mouvements, se substituant parfois aux sociabilités nationalistes ou de voisinage (Tarrius, 2008 ; Helly, 2006). Mais dans le paradigme de la mobilité, celles-ci se substituent presque intégralement aux politiques d'aide sociale. $\mathrm{Au}$ Canada, il existe des droits et une certaine protection sociale pour les travailleurs temporaires, mais c'est généralement par l'obtention du statut de résident permanent que la couverture sociale publique est la plus étendue. Les migrants temporaires dépendent de couvertures privées financées par les employeurs et les travailleurs, sans contrepartie de

8 La secrétaire du Département du Travail et de l'Emploi des Philippines déclarait ainsi que l'« exportation de personnes » constitue la contribution des Philippines au reste du monde. « It is not politically correct to say that you're exporting people... but it's part of globalization and I like to think that countries like ours have that to contribute to the rest of the world » (cité dans Kassam, 2009). 
l'État (Munhak, 2009). Ce sont là des critiques importantes qui font craindre l'apparition de nouvelles stratifications sociales et de nouvelles formes de vulnérabilité. Si la mobilité permet de dépasser les contrôles souverains, elle n'est pas en soi source de droits et de pouvoirs pour les individus, comme certains tentent de le faire reconnaitre (Pécoud et De Guchteneire, 2007).

\section{Références bibliographiques}

ABELLA Manolo (2006) Policies and Best Practices for Management of Temporary Migration, International Symposium on International Migration and Development, 59 p., [en ligne] consulté le 10 mars 2009. URL : http://www.un.org/esa/population/migration/turin/Symposium_Turin files/P03_SYMP_Abella.pdf

ADEPOJU Aderanti (2006) Placing international migration in the context of the 3D's: Demography, Development and Democracy, International Migration, 44 (4), pp. 3-13.

AGUNIAS Dovelyn Rannveig (2009) Guiding the invisible hand: Making migration intermediaries work for development, Human Development Research Paper, 22, UNDP, 95 p., [en ligne] consulté le 15 mai 2009. URL : http://www.migrationpolicy.org/pubs/agunias_HDRP_2009.pdf

ARRIGAGUADA Irma (2009) Feminization of Migration in Latin America: Domestic workers, FOCALPoint, 8 (12), pp. 1-4.

AYDEMIR Abdurrahman et ROBINSON Chris (2006) L'immigration : nouveau visage du Canada, Énoncé de politique, Chambre de Commerce du Canada, $13 \mathrm{p}$.

BASOK Tania (2002) Tortillas and Tomatoes, Montreal \& Kingston, McGill_Queen's University Press, $168 \mathrm{p}$.

BHAGWATI Jagdish (2003) Borders beyond Control, Foreign Affairs, Jan-Feb., pp. 98-104.

BORJAS George (1989) Economic Theory and International Migration, International Migration Review, 23 (3), pp. 457-485.

B.O.R.T.C.E. (2008) Rapport d'étape 2007-2008. Aider les travailleurs formés à l'étranger à réussir au Canada, Ministère des Ressources humaines et Compétences du Canada, Ottawa, 22 p., [en ligne] consulté le 8 avril 2009. URL : http://www.competences.gc.ca/ausujet/rapportetape2007.asp

BOYD Monica, TAYLOR Chris and DELANEY Paul (1982) Temporary Workers in Canada: A multifaceted programme in Temporary Worker Programmes: Mechanisms, Conditions, Consequences, International Migration Review, 24 (4), pp. 929-950.

BREM Maxwell (2006) Les travailleurs migrants au Canada : une revue du Programme des travailleurs saisonniers agricoles du Canada, Dossiers de politiques, Ottawa, Institut Nord-Sud, 18 p.

C.I.C. (2008a) Faits et chiffres 2008. Aperçu de l'immigration, Ottawa, Ministère de la Citoyenneté et de l'Immigration Canada, [en ligne] consulté le 10 avril 2009. URL : http://www.cic.gc.ca/ francais/ressources/statistiques/faits2008/permanents/01.asp

C.I.C. (2008 b) Faits et chiffres. Aperçu de l'immigration. Résidents permanents et temporaires, Ottawa, Ministère de la Citoyenneté et de l'Immigration Canada, [en ligne] consulté le 20 avril 2009. URL : http://www.cic.gc.ca/francais/ressources/statistiques/faits2008/index.asp

C.I.C. (2008c) Rapport annuel au Parlement sur l'immigration, Ottawa, Imprimerie nationale, [en ligne] consulté le 20 avril 2009. URL : http://www.cic.gc.ca/francais/ressources/publications/ rapport-annuel2008/index.asp

C.I.C. (2005) Chiffres pour l'ensemble de l'année 2004, L'Observateur, printemps, Ottawa, 21 p.

COUNIHAN Christopher and MILLER Mark J. (2006) Competing for Global Talent in an Age of Turbulence, in Christiane Kuptsch and Eng Fong Pang Dirs., Competing for Global Talent, Genève, OIT, pp. 259-275.

REMI 2011 (27) 2 pp. 57-75 
CRESSWELL Tim (2006) On the Move. Mobility in the Modern Western World, London, Routledge, $327 \mathrm{p}$.

CUIN Charles-Henry (1993) Les Sociologues et la Mobilité sociale, Paris, PUF, 304 p.

DE VORETZ Don (2006) The Education, Immigration and Emigration of Canada's Highly Skilled Workers in the 21st Century, Research on Immigration and Integration in the Metropolis Working Paper, 06-16, $57 \mathrm{p}$.

DOBROWOLSKY Alexandra (2005) What drives the social investment state in Britain? Ideas and institutions, interests and identities, Annual meeting of the American Political Science Association, Washington DC, $42 \mathrm{p}$.

FAVELL Adrian, FELDBLUM Miriam and SMITH Michael Peter (2008) The Human Face of Global Mobility: A Research Agenda, in Michael Peter Smith and Adrian Favell Eds., The Human Face of Global Mobility, New Brunswick, Transaction Publishers, pp. 1-28.

FINANCES CANADA (2006) Avantage Canada. Un Canada fort dans un monde en évolution, [en ligne] consulté le 20 avril 2009. URL : http://www.fin.gc.ca/ec2006/plan/plc1-fra.asp

FORTES Meyer (1971) Some aspects of migration and mobility in Ghana, Journal of Asian and African Studies, 6 (1), pp. 1-20.

GABRIEL Christina and MACDONALD Laura (2004) The hypermobile, the mobile, and the rest: Patterns of inclusion and exclusion in an emerging North American migration régime, Canadian Journal of Latin American and Caribbean Studies, 29 (57-58), pp. 67-91.

GEYER Gunnar and STRAUBHAAR Thomas (2005) Globalization and loyalty. Who are "we"?, in Max Miller Dir., Worlds of Capitalism. Institutions, Governance and Economic Change in the Era of Globalization, London, Routledge, pp. 268-298.

HELLY Denise (2006) Diaspora : un enjeu politique, un symbole, un concept ?, Espace, Populations, Sociétés, 1, pp. 17-31.

HOLLIFIELD James (2004) The Emerging Migration State, International Migration Review, 38 (3), pp. 885-912.

HMED Choukri et LAURENS Sylvain (2008) Un travail collectif sur la fabrique des catégories et l'invention de l'immigration, Agone, 40, pp. 1-14.

INDUSTRIE CANADA (2008) La mobilité internationale des travailleurs hautement qualifiés : synthèse des principaux résultats et de leurs incidences sur les politiques, [en ligne] consulté le 25 mai 2009. URL : http://www.ic.gc.ca/eic/site/eas-aes.nsf/fra/ra02070.html

JENSON Jane and SAINT-MARTIN Denis (2003) New roots to Social Cohesion? Citizenship and the Social Investment State, Canadian Journal of Sociology, 28 (1), pp. 77-99.

KASSAM Ashifa (2009) Exporting human capital has unexpected costs for the Philippines, FOCALPoint, 8 (12), pp. 14-15, [en ligne] consulté le 27 mai 2009. URL : http://www.focal.ca/ publications/focalpoint/fp0409se/print.asp?lang=e\&article=article6

KAUFMANN Vincent (2002) Re-Thinking Mobility. Contemporary Sociology, Aldershot, Ashgate, $112 \mathrm{p}$.

LARBIOU Benoît (2008) Organiser l'immigration. Sociogenèse d'une politique publique (19101930), Agone, 40, pp. 41-60.

LARBIOU Benoît (2003) Connaître et Traiter l'étranger. Les constructions sociales d'un savoir politique sur l'immigration 1914-1945, Thèse de doctorat, Faculté de droit et de science politique de Montpellier, $880 \mathrm{p}$.

LAVENEX Sandra (2008) The competition state and multilateral liberalization of highly skilled migration, in Michael Peter Smith and Adrian Favell Eds., The Human Face of Global Mobility. International Highly Skilled Migration in Europe, North America and the Asia-Pacific, New Brunswick, Transaction Publishers, pp. 29-52.

MARTINIELLO Marco et SIMON Patrick (2005) Les enjeux de la catégorisation. Rapports de domination et luttes autour de la représentation dans les sociétés post-migratoires, Revue Européenne des Migrations Internationales, 21 (2), pp. 7-18. 
MEYER Antoine and WITKAMP Auke (Eds.) (2008) People on the Move. A Handbook of Selected Terms and Concepts, The Hague, The Hague Process on Refugees and Migration, UNESCO, [en ligne] consulté le 10 mai 2009. URL : http://www.thehagueprocess.org/documents/ THP-UNESCO-HANDBOOK-version-1.pdf

MEYER Jean-Baptiste, KAPLAN David et CHARUM Jorge (2001) Nomadisme des scientifiques et nouvelle géopolitique du savoir, Revue Internationale des Sciences Sociales, 168 (2), pp. 341-354.

MORICE Alain et MICHALON Bénédicte (2008) Les Migrants dans l'agriculture : vers une crise de main-d'œuvre ?, Études rurales, 182, pp. 9-28.

MUNHAK Algacir (2009) Chile: Labour insertion opens dors to citizenship, FOCALPoint, 8 (12), pp. 24-25.

NOIRIEL Gérard (2008) L'Immigration : naissance d'un " problème » (1881-1883), Agone, 40, pp. $15-40$.

O.C.D.E. (2002) La mobilité internationale des travailleurs hautement qualifiés, L'Observateur, Juillet, $7 \mathrm{p}$.

O.I.M. (2008a) La recherche de travail et de travailleurs sera au cœur des mouvements migratoires de ce siècle, selon le rapport 2008 sur l'état de la migration dans le monde, [en ligne] consulté le 15 mai 2009. URL : http://www.iom.int/jahia/Jahia/newsArticleEU/cache/offonce/ lang/fr?entryId=20343

O.I.M. (2008b) Communiqué de presse, 2 décembre, [en ligne] consulté le 23 mai 2009. URL : http://www.iom.int/jahia/Jahia/newsArticleEU/lang/fr/cache/offonce?entryId=203

O.I.M. (2005) International Agenda for Migration Management, Bern Initiative, Geneva, O.I.M., 228 p.

ONSLOW-COLE Julia (2004) Trends in Europe: the Politicisation of UK Immigration Policy, International Legal Practitioner, 29, pp. 201-206.

PÉCOUD Antoine et DE GUCHTENEIRE Paul (2007) Migration Without Borders. Essays on the Free Movement of People, Paris, New York, UNESCO, Berghahn, 294 p.

PERRUCHOUD Richard (2007) Glossaire de la migration, Genève, I.O.M., 98 p.

PICHÉ Victor, PELLETIER Eugénie et EPALE Dina (2006) Identification des obstacles à la ratification de la Convention de l'O.N.U. sur la protection des droits de tous les travailleurs migrants et des membres de leur famille : le cas du Canada, Rapport pour l'UNESCO, section des migrations internationales et des politiques multiculturelles, 25 p., [en ligne] consulté le 10 mai 2009. URL : http://unesdoc.unesco.org/images/0014/001473/147310e.pdf

PREIBISCH Kerry L. (2007) Local Produce, foreign labor: Labor mobility programs and global trade competitiveness in Canada, Rural Sociology, 72 (3), pp. 418-449.

PUTNAM Robert D. (1995) Bowling alone: America's declining social capital, Journal of Democracy, 6 (1), pp. 65-78.

REITZ Jeffrey G. (2005) Tapping immigrants' skills: New directions for Canadian immigration policy in the knowledge economy, IRPP, Choices, 11 (1), pp. 2-15.

RIST Gilbert (2002) Le texte pris aux mots, in Gilbert Rist, Les Mots du Pouvoir. Sens et non-sens de la rhétorique internationale, Paris, PUF, pp. 25-41.

SHAMIR Ronen (2005) Without Borders? Notes on Globalization as a Mobility Regime, Sociological Theory, 23 (2), pp. 197-217.

SHELLER Mimi and URRY John (2006) The new mobilities paradigm, Environment and Planning A, 38, pp. 207-226.

STASIULIS Daiva and BAKAN Abigail (2003) Negotiating Citizenship: Migrant Women in Canada and the Global System, Toronto, University of Toronto Press, 233 p.

TARRIUS Alain (2008) Migration by the poor and economic globalization: transnational migratory flows in Southern Europe, in Christina Gabriel and Hélène Pellerin Dir., Governing International Labour Migration. Current Issues, Challenges and Dilemmas, London, Routledge, pp. 163-181.

WALLACE Claire (2002) Opening and closing borders: migration and mobility in East-Central Europe, Journal of Ethnic and Migration Studies, 28 (4), pp. 603-625. 


\title{
De la migration à la mobilité : changement de paradigme dans la gestion migratoire. Le cas du Canada
}

\author{
Hélène Pellerin
}

\begin{abstract}
Cet article suggère que l'on assiste à l'émergence d'un paradigme de la mobilité, caractérisé par une vision de la migration et de son insertion dans l'économie des pays industrialisés mettant l'accent sur la circulation du capital humain, la présence accrue du privé dans la gestion migratoire et un discours dominant de la mobilité axé sur la recherche de plus grande productivité. Ce paradigme émergeant se substituerait au paradigme de la production des années d'après-guerre. Le cas de la gestion migratoire au Canada est étudié pour explorer les implications de cette transformation.
\end{abstract}

\section{From Migration to Mobility: Paradigm Change in Migration. The Canadian Case}

Hélène Pellerin

This article proposes to explore the emerging mobility paradigm characterized by a specific conception of migration and its insertion in the economy that focus on human capital, the increasing involvement of the private sector in managing migration, and a discourse of mobility directed towards greater productivity. This emerging paradigm, is replacing the production paradigm of the post-war years. The Canadian case of migration management will be used to explore the implications of this transformation.

\section{De la migración hacia la movilidad: cambio de paradigma en la migración. El caso de Canadá}

\author{
Hélène Pellerin
}

Este artículo sugiere que existe un nuevo paradigma en cuanto a la movilidad, caracterizado por la visión de los países industrializados hacia la migración, es decir, la manera en que han logrado incorporarla en la economía, poniendo énfasis sobre la circulación del capital humano; el acrecimiento de la función del sector privado en la gestión migratoria; y el discurso dominante con respecto a la movilidad, que se enfoca en aumentar la productividad. Dicho paradigma emergente sustituye el paradigma de producción destacado en el periodo de posguerra. Se expone el caso de la gestión migratoria en Canadá, con vista a investigar lo que implica esta transformación de la gestión migratoria. 